immeubles habités; mais que dès le mois d'avril I904, la Société a installé dans un coin du parc de X... une guérite spéciale pour le transformateur, avec précision fournie par la Société défenderesse qu'elle a choisi, - pour amener le courant Socte tension dans cette guérite, un câble particulier qui aurait présenté des garanties absolues d'isoliement, même au milieu de l'humidité.

Attendu que S... soutient, à juste titre, que la Société électrique est responsable du déplorable accident dont il s'agit; qu'il résulte, en effet, des documents produits que ladite société a disposé dans le parc de X ... et à la sortie de la petite cabane dans laquelle elle avait placé le transformateur, un câble à tension normale d'environ 3000 volts, à I mètre 22 cent. audessus du sol; que ce câble qui présentait un extraordinaire danger était accessible au personnel de X... et mieux, à toute personne;

Attendu qu'il est bien plaidé, au nom de la Société, que le demandeur devrait avant tout prouver la faute, la négligence et l'imprudence de cette Société; qu'il est bien spécifié que ladite Société a fait tout le possible pour éviter l'accident, puisque l'isolant du câble était, d'après le constructeur, suffisant pour que l'humidité ne soit pas à redouter et que, d'autre part, deux plaquettes avaient été apposées sur les poteaux adducteurs des fils avec l'inscription suivante : "Il y a danger de mort de toucher ces fils $\gg$;

Mais attendu que ces explications restent insuffisantes;

Que la prudence la plus élémentaire exigeait qu'un câble aussi dangereux fut placé hors de toute portêe; que la Société électrique semble même l'avoir reconnu puisqu'il a été déclaré pendant le cours des débats et non dénié par elle, qu'une barrière protectrice avait été placée depuis le jour de l'accident et que le câble avait été enfermé dans une gaîne dépassant la hauteur d'un homme, empêchant ainsi toute atteinte sans forcer la barrière ou grimper sur le poteau;

Attendu que si X... a autorisé la Société à placer une cabane ou guérite dans son parc, il n'est pas douteux pour le Tribunal que la surveillance de cette cabane et du câble qui en était l'accessoire incombait à la Société ; qu'en admettant même que le câble ait pu perdre sa puissance d'isolement par suite des pluies ou des variations atmosphériques, il appartenait à cette Société de prendre les mesures nécessaires pour remédier à cet état de choses; que le danger était connu ou tout au moins prévu, toutes les précautions possibles devaient être prises pour y parer:

Attendu, d'ailleurs, qu'aux termes de l'article $\mathrm{I} 386$, tout propriétaire d'un bâtiment est responsáble des défauts qui y sont inhérents; qu'il faut précisément assimiler aux bâtiments les choses qui en dépendent par incorporation, tels que les mécanismes et les appareils qui en font partie intégrante,

Vu les articles 1382 , r 383 et 1384 du.C. civ.;

Et, attendu qu'il est de principe, que lorsqu'un enfant á été victime d'un accident mortel, les Tribunaux doivent, pour fixer ta réparation due aux parents, apprécier avec le dommage moral, le dommage que leur cause la perte des salaires que gagnait l'enfant et l'assistance qu'ils devaient en espérer pour lavenir ;

Qué, dáns l'espèce, la situation du demandeur serait non seu. fêment très pénible, mais très précaire; que le père pouvait fégitimement compter sur son en fant qui, logé et nourri, gagnait déjà 20 francs par mois et dont les salaires pouvaient être augmentés à brève échéance;

Attendu, cependant, que poùr l'évaluation des dommagesintérêts, le Tribunal doit remarquer que la malheureuse vicfime a commis elle-même une imprudence manifeste; que sächant lire et écrire, cet enfant pouvait remarquer les deux plaqueśdont il a été parlé ci-dessus, et qu'en effet ce n'est pas par simple inattention ni mégarde qu'il a trôlé le câble électrique ;

Vú l'article i 30 du Code de procédure civile ;

Par ces motifs,

Condamne la Société électrique à payer à $\mathrm{S}$... la somme de 2.000 francs, à titre de dommages-intérêts pour les causes dont Il s'agit, avec les interêts, à compter du jour de la demande;

La condamne aux dépens.

\section{Nouveau type de Locomotive électrique}

Le 12 novembre Igo4, on a expérimenté une locomotive électrique d'un type tout nouveau, construite par la General Electric Company et 'par l'American Locomotive Company pour le service des trains râpidés de la ligne $\mathrm{New}$ York-Croton et du trónçon de Harlem que la Nèw York Central and Hudson River Räilroad Companýy á récemment équipés élèctriquement.

La disposition des moteurs électriques dè cette voitùre diffère totalement de celle qui est généralement adoptée dans les types ordinaires. Aussi nous a-t-il paru intéressant d'en donner ici une description d'après l'Ingegneria Ferroviaria et le Railway Age.

Cette locomotive est supportée par six paires de roues dont quatre sont motrices. Le poids de la voiture se transmet au châssis et aux essieux au moyen de balanciers en acier forgé, ainsi que cela se fait d'habitude pour répartir la charge d'une façon rationnelle.

La caractéristique principale de cette locomotive est la suivante : les induits des moteurs sont bobinés directement sur les essieux moteurs et le circuit magnétique inducteur est constitué par le chassis de la voiture. On réalise ainsi la disposition la plus simple qu'il paraisse possible d'imaginer. La figure ci-jointe permet de se rendre facilement compte du système.

Les tôles des induits sont directement calées sur les essieux moteurs, et l'enroulement induit est bobiné dans les rainures ménagées dans ces tôles. Les faces polaires sont légèrement concaves dans la partie centrale et: l'entrefer varie de trois-quarts de pouce à un pouce (19 à $25 \mathrm{mms}$ ). Le plus grand déplacement vertical que puisse prendre l'induit par rapport aux pièces polaires est de trois pouces (75 mms); ce mouvement vertical est limité par des pièces métalliques solidaires de la suspension. Les porte-balais sont fixés sur le châssis par des isolateurs montés sur des étriers, de manière que leurs positions respectives soient indépendantes des oscillations du véhicule, et leurs axes sont réglables pour pouvoir compenser l'usure des balais produite par le frottement de ceux-ci sur le collecteur.

Le changement d'un induit grillé ne paraît pas plus difficile que dans les autres itypes de commande électrique; cela revient à changer un axe dans ùie locomotive ordinaire.

Le courant nécessaire est normalement capté sur un troisième rail au moyen de huit frotteurs, dont quatre de chaque côté de la locomotive. Cependant, dains les stations importantes, où les croisements sont très nombreux, la prise de courant se fait par le haut, au moyen de frotteurs spéciaux que l'on peut voir représentés sur la toiture de la locomotive. Les appareils qui supportent les frotteurs aériens sont amovibles, on peut les monter ou les abaisser à volonté, de l'intérieur de la locomotive, au moyen d'air comprimé pris sur la commande des freins..

La cabine de commande de la locomotive se trouve au centre de la voiture Là sont-les contrôleurs; les rhéostats, les appareils de mesure, voltmètres, ampèremètres, les coupe-circuits, les mécanismes de commande des freins', des sablières, des sifflets, etc. Tous cess appareils, qui sónt en double pour la marche avant et pour la marche arrière, sont du type Sprague-General Electric.

On peut réaliser trois montages différents pour la commande des moteurs électriques: Io Les quatre moteurs sont en série (petite vitesse); $2^{\circ}$ Les moteurs sont en série 
deux par deux et les deux groupes ainsi formés sont en parallèle (vitesse moyenne); $3^{\circ}$ Les quatre moteurs sont en parallèle (grande vitesse).

Le compresseur d'air est constitué par deux cylindres verticaux actionnés par deux moteurs série à 600 volts. Il peut débiter 75 pieds cubes $\left(\mathrm{o}^{3} \mathrm{o}^{2} 83\right)$ par minute. Un dispositif automatique met le moteur en circuit ou hors circuit selon la pression de l'air.

La caisse de la locomotive a été encore prévue pour pouvoir disposer une petite chaudière chauffée à la benzine ou au pétrole pour le chauffage des trains. dans la cabine centrale, inscrivaient les diagrammes des efforts de traction et de la puissance consommée, permet: tant ainsi de se rendre compte comment se comportaitla locomotive.

La plus grande vitesse atteinte en remorquant le train de 8 wagons a été de 63 milles à l'heure, soit roo kilomètres; en remorquant celui de 4 wagons elle a été de 72 milles, soit I I 6 kilomètres. Il est à noter que ces vitesses étaient les maxima que l'on s'était fixé, à cause de la faible longueur de la ligne affectée aux essais.

Les diagrammes relevés ont indiqué qu'avec le train de

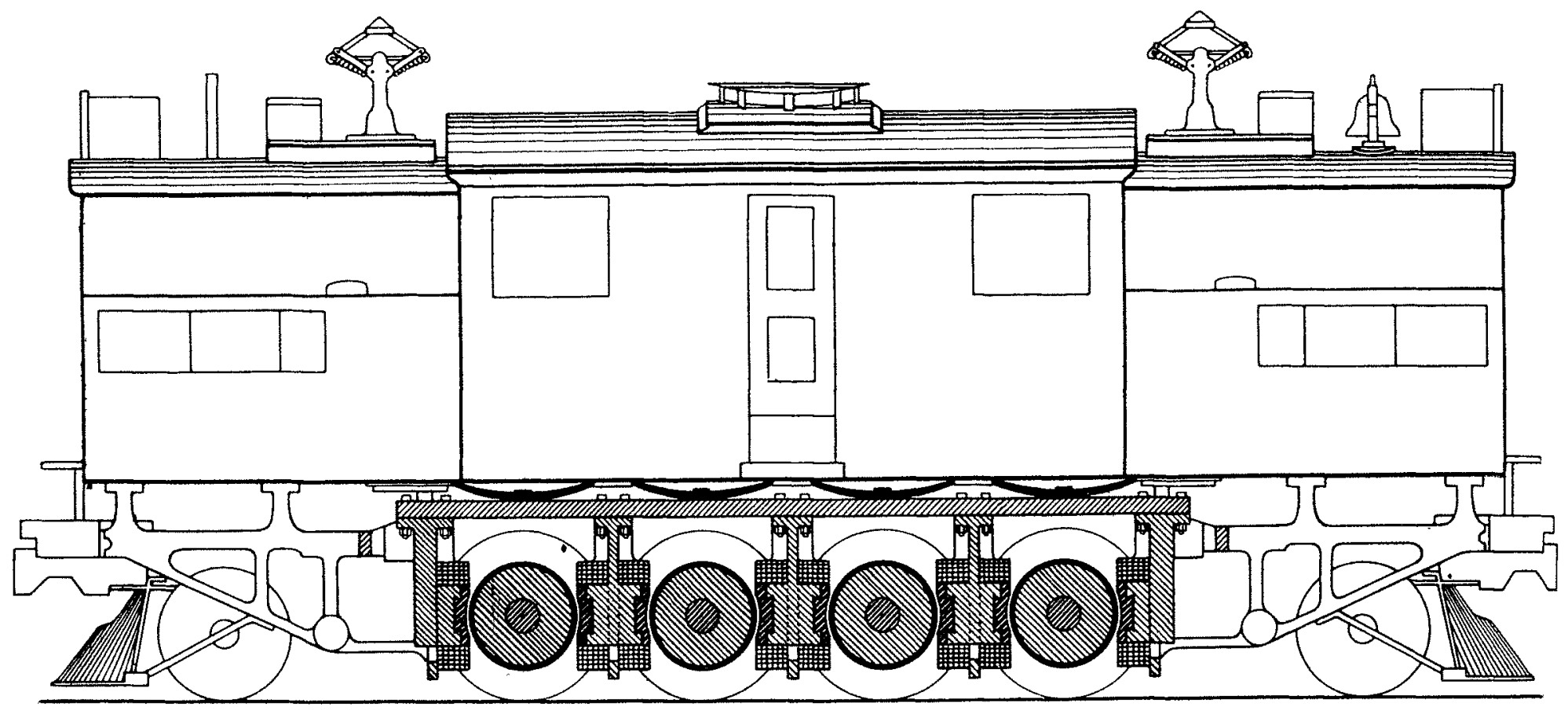

SCHÉMA GÉNÉRAL DE LA LOCOMOTIVE

Voici quelles sont les caractéristiques principales de cette locomotive :

Nombre total de roues............... 12

Nombre de roues motrices............. 8

Poids total de la locomotive.... en tonnes-livres 95

Poids correspt sur les roues motrices 》 $\quad 69$

Longuețr du châssis magnétique..... en pieds 13

Empattement total des roues........ \ $\quad 27$

Longueur totale de la voiture....... » 37

Largeur maximum de la voiture..... 》 10

Hauteur dı toit de la voiture....... »

Diamètre des roues motrices........ en pouces

Diamėtre des autres roues motrices... \ 36

Diamètre des axes moteurs........ "
Puissance normale de la locomotive... en HP 2200

Puissance maximum développée..... \ 3000

Effort normal de traction .......... en livres 20400

Effort maximum au départ........ 》 32000

Vitesse à l'heure d'un train de 500 tonnes en milles 60

Voltage .................. en volts 600

Courant normal, à pleine charge.... en ampères 3070

Courant maximum à pleine charge... 》 4300

Nombre des moteurs (GE, 84 A).... 》

Puissance de chaque moteur...... en HP 50

Les essais ont été faits sur une ligne spéciale, soit en remorquant un train de 8 wagons et du poids de 336 tonnes-livres ( $34 \mathrm{I}$ ooc $\mathrm{kgs}$ ), non compris la locomotive, soit en remorquant un train de 4 wagons et du poids de 170 tonnes-livres ( 173 ooo $\mathrm{kgs}$ ), toujours sans la locomotive.'

Pendant ces essais, des appareils enregistreurs, placés $(\mathrm{kms}=96)$
8 wagons, pesant avec la locomotive $43 \mathrm{I}$ tonnes-livres (438 00o kgs), on a pu atteindre la vitesse de 30 milles ( 48 kilomètres) en 60 secondes, ce qui correspond à uné accélération de $o^{m_{2}} 6$ à la seconde. A certains moments cette accélération a même atteint $\mathrm{o}^{\mathrm{m}} \mathbf{2} 6$ à la seconde, avế un effort de traction de 27000 livres ( $12200 \mathrm{kgs}$ ) à. la péri: phérie des roues motrices.

Avec le train de 4 wagons, dont le poids total atteignait, avec la locomotive, 265 tonnes-livres ( $269000 \mathrm{kgs})$, la con: sommation de courant a été, à un certain moment, de 4200 ampères, avec un effort de traction de 3 I 000 livres (14 ooo kgs) à la périphérie des roues motrices et avec un coefficient de traction de 20 pour 100 du poids sur ces mêmes roues. La vitesse de 30 milles à l'heure $(48 \mathrm{kms})^{\text {ḋ }}$ été atteinte en 37 secondes et demie avec un effort de traction correspondant de 22000 livres ( $9960 \mathrm{kgs})$. La plus grande consommation de courant a été de 4200 ampères sols 460 volts aux bornes des moteurs. dont la puissance meslu rable sur les roues était de 2200 chevaux. Si le voltage avait été maintenu à 600 volts, les moteurs auraient p甘 développer 2870 chevaux avec un coefficient de traction de 22,5 pour I oo du poids sur les roues motrices, dépassant ainsi tout ce qu'on avait pu obtenir avec les locomotives ? vapeur construites jusqu'à ce jour.

Les diagrammes relevés ont de plus permis de constatet le haut rendement des moteurs électriques qui a puattein dre jusqu'à $9^{3}$ pour soo, supérieur de 4 pour too aux plus hauts rendements obtenus avec des moteurs à engrenagesi et de plus, alors que le rendement de ceux-ci diminut 
lorsque la vitesse augmente, dans le type de locomotive essayée le rendement se maintient à peu près constant.

Le courant nécessaire à ces expériences était fourni par une sous-station, transformant, en courant continu, le conrant alternatif produit par un alternateur générateur directement accouplé à une turbine à vapeur Curtis de la station centrale de Schenectady, où se trouvent les usines de la General Electric Co.

Ce type de locomotive a été étudié, non pour atteindre de très grandes vitesses, mais pour pouvoir obtenir une accélération de beaucoup supérieure à celle des locomotives à vapeur. Les premiers résultats obtenus se sont montrés, sur ce point, assez satisfaisants. $\quad$ L. R.

\section{LE MOIS HYDRO-ÉLECTRIQUE}

\section{ACADÉMIE DES SCIENCES}

\section{MÉGANIQUE ET ELECTRIGITÉ}

Champ magnétique auquel est soumis un corps en mouvement dans un champ électrique. - Note de M. Pellat, séance du 23 janvier.

Un corps animé d'une grande vitesse $y$ dans un champ électrique d'intensité $\Phi$ est soumis par là même à un champ magnétique dont la direction est normale au plan contenant la direction de la vitesse et celle du champ électrique, dont le sens est la droite d'un observateur disposé de facun que le mouvement aille de ses pieds à sa tête et qui regarderait dans le sens du champ électrique, et dont lintensité est donnée par:

$$
\mathscr{H}=K \Phi \nu \sin \alpha
$$

en appelant $\alpha$ l'angle de la vitesse et du champ électrique et $K$ le pouvoir inducteur spécitique du milieu, cette relation étant exacte dans l'un et l'autre système d'unités électriques.

Pour établir cette proposition, considérons un condensateur à armatures planes parallèles et indéfinıes, chargé et présentant une densité uniforme $\sigma$ sur ses faces en regard. Pour la commodité du langage, prenons ces armatures verticales. Supposons qu'elles se déplacent, en emportant leur charge, avec une grande vitesse $y$, dans leur propre plan, suivant une direction horizontale. Pour tout point fixe placé entre les armatures, 1 en résultera un champ magnétique, car chaque bande' horizontale des armatures en mouvement produit l'effet d'un courant électrique; il est facile de voir que les deux armatures produisent des champs magnétiques de même sens, que le champ magnétique résultant est uniforme, que sa direction est verticale, son sens donné par la règle d'Ampère et son intensité par :

$$
\mathscr{U}=4 \pi \sigma \nu
$$

Si nous supposions maintenant un déplacement des armatures dans une direction normale à celles-ci, les considerations de symétrie montrent immédiatement que le champ magnétique en $P$ serait nul. On déduit de ce qui précède que, si le déplacement des armatures a lieu dans une direction faisant un angle $\alpha$ avec les lignes de force du champ électrique et une vitesse $y$, l'intensité du champ magnétique est donnée par:

$$
\mathscr{H}=4 \pi \sigma \nu \sin \alpha
$$

La relation entre l'intensité $\Phi$ du champ électrique et la densité $\sigma$ sur les armatures est $K \Phi=4 \pi \sigma$; en remplaçant il vient :

$$
\mathscr{H}=K \Phi \nu \sin \alpha
$$

Remarquons maintenant que, en vertu du principe d'action de milien, l'effet sera le même si le point $P$ considéré a le même moude ment relatif par rapport aux lignes de force d'un champ électrique trique intensité, quelle que soit la manière dont ce champ élecrelatif est obtenu et quelle que soit la manière dont le mouvement electrique produit. En particulier, si les lignes de force du champ duira pour sont immobiles et le point $P$ en mouvement, il se protant) un ce point (portion d'un aimant, d'un solénoîde, d'un coude cette note. magnétique conformément à l'énoncé qui est en tête
On voit que la relation ( 1 ) a même forme que la relation qui donne l'intensité du champ électrique agissant sur un point mobile dont la trajectoire coupe sous un angle $\alpha$ les lignes de force d'un champ magnétique : il n'y a qu'à permuter $\mathcal{K}$ et $\Phi$ et à changer $K$ en $\mu$ (perméabilité magnétique) dans les formules, à permuter " magnétique " et "électrique "dans l'énoncé et à changer "droite " en " gauche $\triangleright$. Ainsi le phénomène dont la loi est donnée en tête de cet article est le phénomène réciproque de l'induction électromagnétique.

$\mathrm{Si}$, dans la relation (I), nous employons les unités électromagnétiques, on a $K=\frac{k}{U^{2}}$, en désignant par $k$ la constante diélectrique du milieu (l'unité dans le cas du vide) et par $U$ le rapport des unités $\left(U=3.10^{10}\right)$; de façon que la relation devient :

$$
\mathscr{H}=\frac{k \Phi \nu \sin \alpha}{U^{2}}
$$

Il n'est pas difficile d'obtenir pour $\frac{\Phi}{U}$ une valeur de plusieurs unités ; mais pour que $\mathcal{J}$ soit notable, il faut encore que $\frac{\nu}{U}$ ne soit pas trop inférieur à l'unité, c'est-à-dire que $y$ ne soit pas trop éloigné de la vitesse de la lumière. Ce ne sont guere que les corpuscules lancés par une cathode ou par un corps radioactif qui peuvent être dans ce cas.

M. Villard a montré $\left(^{*}\right)$ que les rayons magnéto-cathodiques découverts par M. A. Broca subissent une déviation en coupant les

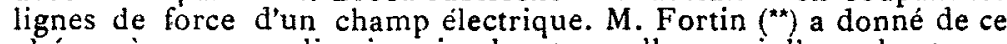
phénomène une explication simple et excellente, si l'on admet que ces rayons sont formés par les mêmes corpuscules que les rayons cathodiques ordinaires; dans ce cas, le phénomène que nous venons d'exposer ne donne naissance qu'à un terme correctif pour la formule de déviation établie par $M$. Fortin. Mais si, conformément à une expérience de $M$. Villard, les rayons magnéto-cathodiques ne transportent avec eux qu'une charge plus faible que celle des rayons cathodiques, l'explication de M. Fortin ne convient plus. Peut-être, peut-on alors expliquer le phénomène de la déviation rien que par la composition du champ magnétique produit par le mouvement dans le champ électrique de ce qui constitue les rayons magnétocathodiques avec le champ magnétique préexistant (celui de l'électroaimant) : ces deux champs magnétiques, à angle droit, donnent par leur composition un champ oblique que doit suivre le rayon magnéto-cathodique, d'après sa propriété fondamentale. Le sens de la déviation due à cette cause est bien conforme à celui trouvé par M. Villard. Quant à l'ordre de grandeur, il faudrait connaître les intensités des champs électriques et magnétiques employés par ce physicien pour voir si l'explication ne nécessite pas des valeurs trop grandes pour la vitesse des rayons magnéto-cathodiques. Tout ce que l'on peut dire, c'est qu'avec des intensités de champ facilement réalisables et des vitesses d'un ordre de grandeur très acceptable, on obtiendrait de ce chef des déviations non seulement visibles, mais bien mesurables.

Sur un nouvel embrayage. - Note de M. HÉrisson, séance du 6 février.

La puissance et la progressivité sont les qualités essentielles que l'on recherche dans, les embrayages. La progressivité n'est pas la moins importante, car, en évitant toute brusquerie dans la transmission du mouvement, clle donne une grande douceur à la mise en marche, assure la conservation du mécanisme et permet l'emploi d'organes plus légers.

Jusqu'ici la progressivité des embrayages purement mécaniques n'a été réalisée que par un seul moyen : en les réglant pour une puissance très légèrement supérieure à celle du moteur, en sorte qu'ils patinent pendant la période de misc en route et absorbent ainsi, par un travall de frottement, un partie de la puissance du moteur, ce qui permet l'entraînement progressif de la machine conduite. Ce moyen expose l'embrayage à patiner d'une manière permanente.

Le but que l'auteur a poursuivi a été d'obtenir des embrayages absolument progressifs, quelle que soit leur puissance, et il a réalisé cette conception par le dispositif mécanique suivant

Soit une cuvette entraînée par un moteur et tournant folle sur un arbre relié à la machine conduite; le corps de l'embrayage consiste en un support portant deux parties symétriques constituées chacune par un patin pouvant se déplacer du centre vers la circonférence; ce patin est appuyé contre la paroi intérieure de la cuvette par un levier, mobile dans le plan de l'axe du patin et de l'axe de l'arbre, levier articulé sur le support en un point voisin du fond de la cuvette. Supposons que le mouvement de ce levier soit obtenu par l'effet d'un coin soulevant son extrémité labre en glissant sur l'arbre, quand il est poussé du côté du support, par un collier sur lequel agit le levier d'embrayage. C'est là un moyen de serrage non réversible. Supposons, de plus,

(*) Villard, Comptes rendus, t. CXXXVIII, 1904, p. 6408.

(**) Forțin, Comptes rendus, t. CXXXVIII, t904, p. 3594 . 